\title{
Clustering Students based on Their Annotations of a Digital Text
}

\author{
Keith Ying ${ }^{1}$, Maiga Chang ${ }^{2}$, Andrew F. Chiarella ${ }^{3}$, Kinshuk ${ }^{2}$, Jia-Sheng Heh ${ }^{1}$ \\ ${ }^{1}$ Department of Information and Computer Engineering, Chung-Yuan Christian University, Chung-Li, Taiwan \\ ${ }^{2}$ School of Computing and Information Systems, Athabasca University, Athabasca, Canada \\ ${ }^{3}$ Centre for Social Sciences, Athabasca University, Athabasca, Canada \\ keith70167@hotmail.com,maiga@ms2.hinet.net, andrewc@athabascau.ca, kinshuk@athabascau.ca, \\ jsheh@ice.cycu.edu.tw
}

\begin{abstract}
Students often annotate texts they are reading using highlighting, underlining, and written comments and marks in the margins of the text. These may serve various functions and will reflect each student's goals and understanding of the text. This research proposes two simple biology-inspired approaches to represent the patterns of student annotations and to cluster students based on the similarity between their annotations; the annotations produced were simple highlighting. To verify the effectiveness of the proposed approaches, the research compared the processing speed of these approaches with generic hierarchical clustering algorithm implemented in Matlab and compared the accuracy of the clusters with the clusters created by human raters. The results show that both of the proposed approaches are more efficient and accurate than the generic hierarchical clustering algorithm. The proposed methodology can be implemented as an add-on to existing learning management systems and e-book readers, to automatically offer the students important notes and annotations conducted by others (either peers or students in the past) who have similar annotation behaviour pattern and style to the students.
\end{abstract}

Keywords-Annotation; Biology-inspired; Chromosome; Patterns; Clustering

\section{INTRODUCTION}

Annotation is often a useful tool that readers use to help them to enhance their learning from text [2]. In one common form of annotation, students read textbooks and highlight the keywords they believe important in order to make their learning more efficient. Due to the popularity of Internet, many researchers have now developed web-based annotation tools [11][25] or reading software that supports such annotation.

Annotations made by different students are usually not same and can be considered as a behavior pattern which represents each student. For this reason, this research converts the highlights made by a student into the bitstring chromosome and designs two approaches to measure the similarity of two chromosomes. If students have similar highlights on the text, then they may be clustered into the same group. The students in the same cluster may have similar thoughts on the text or similar learning strategies for learning.

The annotation data for highlights on a text is a long data sequence of 0 's and 1's. This research proposes two chromosome-based approaches to transform the students' highlights on the text. We then use those to locate and quantify chromosome differences between students and cluster students with similar chromosomes. In order to assess the performance of the proposed chromosome-based approaches, we first compare the processing time used by each approach with that used by the generic hierarchical clustering method (implemented in Matlab). Furthermore, we check the accuracy rate of each approach by comparing them clusters identified by human raters.

The remainder of the paper is organized as follows: Section II reviews the literature on annotation and clustering approaches. Section III discusses the two proposed approaches; how to convert a student's highlights to a chromosome and how to measure the difference between two chromosomes? Section IV describes the method of clustering chromosomes. Section V shows the evaluation results. Section VI provides a conclusion and discusses possible future research directions.

\section{ANNOTATION AND CLUSTERING}

Annotation can help students read and learn. According to Marshall (1997), there are telegraphic, or implicit, and explicit annotations [18]. The implicit annotations (e.g., highlighting and underlining) contain meanings that generally only the original annotator could know. The explicit annotations (e.g., notes taking and drawings) have more clearly stated meanings that other readers may also understand. In some early research, it was found that students usually preferred to make annotations directly on textbooks or notebooks instead of making electronic annotations [1][20].

As the Internet has become more popular, digital and electronic documents are widely used and accepted by students. Making annotations on electronic documents for learning has gradually become more attractive to students [2]. There are many tools for making annotation on electronic and on-line documents today: Kurhila and colleagues (2003) develop an annotation system called EDUCOSM for web-based learning [15]; Rau and colleagues (2004) develop Web-based Annotation Tools (WATs) for students making annotations directly on the web pages and sharing their annotations with others [22]; Yeh and Lo (2009) develop Online Annotator for EFL Writing which allows teachers to mark errors and to do corrections on online documents and students can receive the corrective feedback from teachers in the online composition class [25]; Chao and colleagues (2010) develop a cross-media annotation system [5]; and, 
Chiarella and colleagues design a reading application (CoREAD) that supports simple highlighting annotations and, in addition, adds social text signals to the text by aggregating the annotations of the readers [6][7].

Some research has been conducted to assess the effectiveness such tools have for improving students' learning performance. Chang and colleagues (2006) have found that annotating learning materials is an effective learning strategy for student's reading comprehension ability [4]; Hwang and colleagues (2007) have found that the student's learning performance does improve in both individual learning and group learning scenarios [12]; and, Hwang and Hsu (2011) have found that pre-reading and annotation sharing have positive impact on student's learning [11].

Clustering is a means used to divide a data set into subsets. The items in a subset are similar or have the same features as the other items [8][24]. Many clustering methods exist. Two well-known clustering methods are Hierarchical Clustering and Partitioning Clustering.

In Hierarchical Clustering a hierarchical tree is constructed that presents the clusters in a tree structure called a dendrogram [3][14]. In the hierarchical tree, the root is a cluster which contains all items from the data set and the leaves are the individual items. Figure 1(a) shows a data set with six items and Figure 1(b) shows a possible hierarchical tree for the data set. Different hierarchical trees can be established with different similarity measurement approaches.

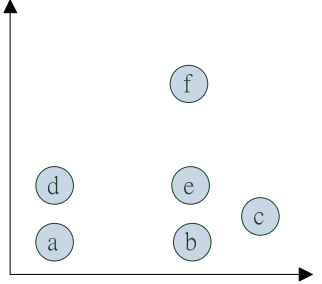

(a) Data Set

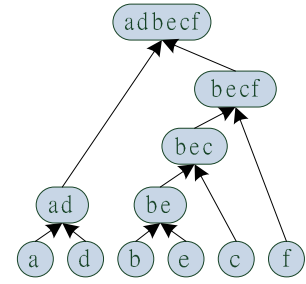

(b) Hierarchical tree
Figure 1.

Partitioning Clustering is different from Hierarchical Clustering as it divides the data set into subsets by predefined parameters such as the number of clusters and the size of clusters [8]. K-means Clustering is one of the common partitioning clustering methods [9][16]: the $\mathrm{K}$ represents the number of clusters. The first step of Kmeans Clustering is to generate $\mathrm{K}$ clusters for the data set by randomly choosing $\mathrm{K}$ items as the center of the clusters.

Take Figure 1(a) as example, supposed $\mathrm{K}$ is set to 2 and the method randomly chooses items "a" and "e" as the cluster centers. The method then clusters items into the two clusters using a distance calculation shown in Figure 2(a). The two clusters and its items are $\{a, d\}$ and $\{e, b, c$, $\mathrm{f}\}$. The method then calculates the new center of the clusters as Figure 2(b) shows. The method stops when it cannot find any further item exchange happens among clusters, which means, items in a cluster have minimum distances to the cluster center compared with the distance to other cluster centers.

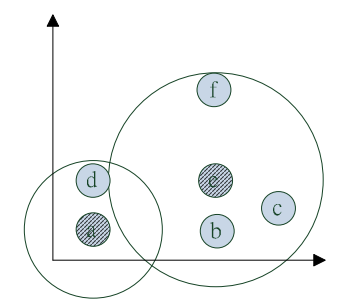

(a) Random choose data as center

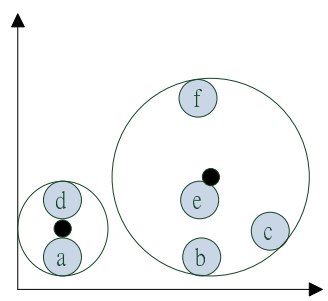

(b) Clusters and its centers
Figure 2.

Therefore, the main purpose of this research is to clustering students according to their annotation behaviors. Furthermore, giving teachers the cluster results so teachers can assist students whose annotations have different focus or miss cover the most important concepts that the particular text is talking; also, giving students feedback such additional notes created by their fellow students of same cluster and supplemental materials that they may need to or may want to know.

\section{CHROMOSOME-BASED HightLIGHT PATTERN AND COMPARISONS}

This research treats a student's implicit annotations (i.e., highlights added to a digital text) as a chromosome; as such, the chromosome can represent the student. The data analyzed was produced by 40 undergraduate students who read a text about the Flynn Effect [19] and highlighted sections of text they determined were important for the task of writing a summary of that text [6][7]. The text was 2894 words long and so each "chromosome" has a length of 2894 bits.

Figure 3(a) shows a sentence from the original text. Figure 3(b) shows the highlights made by one student.

\begin{tabular}{|l|l|}
\hline $\begin{array}{l}\text { Some tests consist only of } \\
\text { abstract-reasoning } \\
\text { problems, and others }\end{array}$ & $\begin{array}{l}\text { Some tests consist only of } \\
\text { abstract-reasoning } \\
\text { focus on such special }\end{array}$ \\
problems, and others \\
fompetencies as & compen such special \\
arithmetic, spatial & arithmetic, spatial \\
imagery, reading, & imagery, reading, \\
vocabulary, memory or & vocabulary, memory or \\
general knowledge. & general knowledge. \\
\hline
\end{tabular}

(b) Highlighted Text

Figure 3.

Each word's highlight status can be represented by 0 (no highlight) and 1 (has been highlighted) as Figure 4 shows, so a bit-string chromosome can be retrieved.

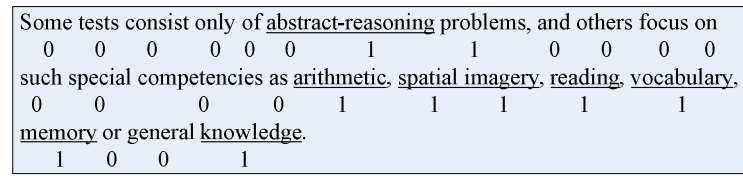

Figure 4 .

Transformed annotation data.

This research develops two basic approaches, the standard approach and the quantitative approach, to 
measure the similarity of chromosomes as well as to cluster chromosomes.

In the standard approach we use one chromosome as the benchmark and measure how different the other chromosomes are to it. As Figure 5 shows, there are four differences between the chromosomes of User \#1 and User \#2; differences occur at positions 2, 7, 11 and 12.

\begin{tabular}{|l|l|}
\hline User \#1 & $\underline{0} 1 \underline{10010 \underline{111} 10 \underline{11011110001}}$ \\
\hline User \#2 & $\underline{0} 0 \underline{1001} \underline{11101} \underline{11011110001}$ \\
\hline
\end{tabular}

Figure 5. Chromosomes represent User \#1 and User \#2.

We assign each difference a $2^{\mathrm{i}-1}$ value, where the parameter i means the position of the difference occurs; in this example, the assigned difference values are $2^{(2-1)}, 2^{(7-1)}$, $2^{(11-1)}$ and $2^{(12-1)}$. The total difference value between User $\# 1$ and User \#2 is $2^{1}+2^{6}+2^{10}+2^{11}=3,138$.

Figure 6 shows another example for User \#1 and User \#3. In this example, the assigned difference values are $2^{(12-}$ 1) $2^{(14-1)}, 2^{(15-1)}, 2^{(23-1)}$ and total difference value is $2^{11}+2^{13}+2^{14}+2^{22}=4,220,928$.

\begin{tabular}{|l|l|}
\hline User \#1 & $\underline{01100101111011011110001}$ \\
\hline User \#3 & $\underline{01100101111} 1 \underline{1011110000}$ \\
\hline
\end{tabular}

Figure 6.

Chromosomes represent User \#1 and User \#3.

Although four differences occurred in both examples the similarity (i.e., the difference value) between the benchmark chromosome and comparison chromosome is quite different. Since the standard approach weights each difference by position the total difference value for pairs of chromosomes with the same number of differences can differ by a large amount; this is especially true when a chromosome's length is large.

With the quantitative approach, we attempt to correct the abovementioned drawback. The quantitative approach also uses $2^{\mathrm{i}-1}$ to calculate a difference value, but the parameter $\mathrm{i}$ here indicates the number of the difference instead of the position where each difference occurs. Using Figure 5 as example, there are four differences occurring at positions 2, 7, 11, and 12. However, the assigned difference value for the difference occurring at position 11 is $2^{(3-1)}$ this time instead of $2^{(11-1)}$, because it is the third difference. Under such circumstance, the total difference value between User \#1 and User \#2 is $2^{0}+2^{1}+2^{2}+2^{3}=15$ if we use the quantitative approach to compare the two chromosomes.

Although the quantitative approach may have undistinguishable cases happened when two chromosomes have the same amount of differences occurred at different positions compared with the same benchmark chromosome, the chromosomes may be considered to categorize into the same cluster in some senses. For instance, given a lengthy benchmark chromosome (e.g., a 200-word paragraph), a few chromosomes have only couple of differences compared to the benchmark chromosome could be clustered together if all other chromosomes have a lot of differences compared to the benchmark chromosome.

\section{Clustering Algorithm}

For student clustering, this research considers both Kmean partitioning clustering and hierarchical clustering methods to cluster the chromosomes by mapping the chromosomes to a two-dimensional plane as Figure 7 and 8 show. To map the chromosomes to a two-dimensional plane according to their difference values to the benchmark chromosome, this research divides a chromosome into two parts and uses each part's difference value as the $\mathrm{x}-\mathrm{y}$ coordinates.

First, we take User \#1 as the benchmark chromosome to calculate the coordinates. Assuming User \#1's coordinates is $(0,0)$, then we can have User \#2's coordinates is $(3,138,0)$ according to the difference value; User \#3's coordinates is $(0,0)$; and, User \#4's coordinates is $(1,1,030)$. For bottom up clustering, each user represents a single user cluster at very beginning. After that, a new cluster $\mathrm{C}_{5}$ can be found by combining cluster $\mathrm{C}_{1}$ and $\mathrm{C}_{3}$ together. The coordinates of cluster $\mathrm{C}_{5}$ 's center is still $(0,0)$ due to both $C_{1}$ and $C_{3}$ locate at same place. We can the merge cluster $C_{5}$ with $C_{4}$ and have a larger cluster $\mathrm{C}_{6}$ with center coordinates $(0.5,515)$. At the end, the cluster $\mathrm{C}_{2}$ and $\mathrm{C}_{6}$ are merged and have a root cluster $\mathrm{C}_{7}$ with center coordinates $(1,569.25,257.5)$. Figure 9 shows the hierarchical tree for the standard approach results.

\begin{tabular}{|c|c|c|}
\hline & $\underline{\mathbf{x}}$ & $\mathbf{y}$ \\
\hline User \#1 & $01 \underline{1001011110}$ & $\underline{11011110001}$ \\
\hline User \#2 & $00 \underline{10011} \underline{11101}$ & $\underline{110 \underline{111000} 1}$ \\
\hline User \#3 & $01 \underline{10010 \underline{111} 10}$ & $\underline{110 \underline{111000} 1}$ \\
\hline User \#4 & $11 \underline{1001011110}$ & $\underline{10111110000}$ \\
\hline
\end{tabular}

Figure 7. Chromosomes representing Users \#1 to \#4.

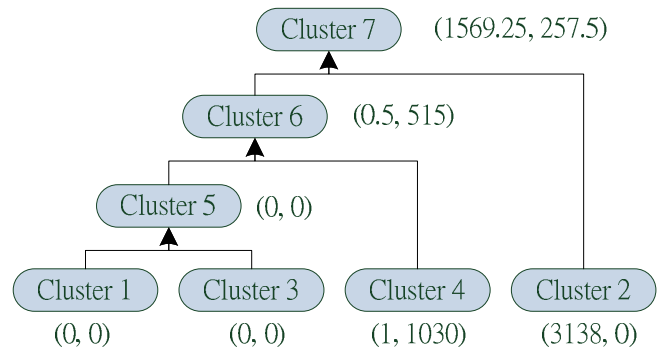

Figure 8. Hierarchical tree of standard approach.

For the quantitative approach results, the process is same. We still take User \#1 as the benchmark chromosome to calculate coordinates. Given User \#1's coordinates is $(0$, $0)$, we can have User \#2's coordinates is $(15,0)$; User \#3's coordinates is $(0,0)$; and, User \#4's coordinates is $(1,3)$. For bottom up clustering, the generated hierarchical tree set is quite similar to the standard approach results as Figure 10 shows. The differences are the coordinates of the cluster centers, for instances, cluster $\mathrm{C}_{6}$ center's coordinates is $(0.5,1.5)$ and cluster $\mathrm{C}_{7}$ center's coordinates is $(7.75,0.75)$. 


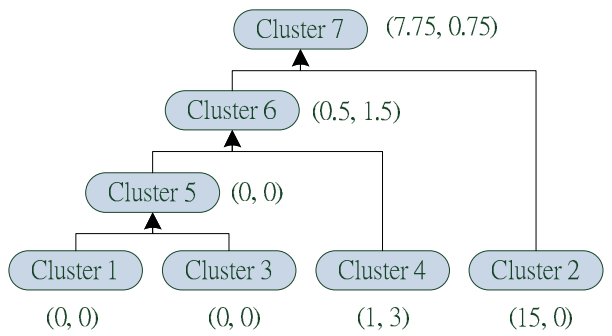

Figure 9

Hierarchical tree of quantitative approach.

\section{Evaluation}

To evaluate the proposed chromosome-based clustering approaches two performance indicators were used: speed (i.e., how fast the approach can process the data) and performance (i.e., how well the task is performed).

First, we implement generic hierarchical clustering method with pdist, linkage, and dendrogram functions in Matlab. We then compared the processing time that different approaches took to cluster the 40 students' annotations. Table I lists the processing times for each algorithm.

TABLE I. TIME COST

\begin{tabular}{|c|c|c|c|c|}
\hline & Standard & Quantitative & $\begin{array}{c}\text { Hierarchical } \\
\text { Clustering }\end{array}$ & $\begin{array}{c}\text { Human } \\
\text { Rater }\end{array}$ \\
\hline $\begin{array}{c}\text { mapping } \\
(\mathrm{ms})\end{array}$ & 5.74 & $\underline{\mathbf{5 . 0 0}}$ & N/A $^{*}$ & N/A $^{*}$ \\
\hline $\begin{array}{c}\text { clustering } \\
(\mathrm{ms})\end{array}$ & 24.21 & $\underline{\mathbf{2 2 . 4 3}}$ & 28.43 & 263,690 \\
\hline Total (ms) & 29.95 & $\underline{\mathbf{2 7 . 4 3}}$ & 28.43 & 263,690 \\
\hline
\end{tabular}

*. for the generic hierarchical clustering and human clusters, there is no additional step of mapping the user's chromosome to two-dimensional plane for calculating the distances from other users' chromosomes for the preparation of partitioning clustering.

Human rater clustering was the slowest approach. The quantitative approach, on the other hand, is the fastest approach. Nevertheless, both of the standard and the quantitative approach take less time for doing clustering than the generic hierarchical clustering approach. With mapping and clustering times combined, the total time for the three approaches is approximately the same.

For the performance evaluation, we use human rated clusters as the benchmark or ideal outcome. The output of the three different approaches (i.e., the two proposed chromosome-based approaches and the generic hierarchical clustering Matlab approach) were therefore compared with the human rated clusters to determine which performed the best.

Two widely used measures for evaluating the performance of such tasks from the information retrieval research field are precision and recall. The modified definition of the precision measure for this research is

precision $=\frac{\mid \text { manual_cluster_users }|\cap| \text { approach_cluster_users } \mid}{\mid \text { approach_cluster_users } \mid} \cdot(1)$

From the definition, we see that precision means the accuracy of an output of an approach. As an example, if there are ten users in the proposed cluster classified by approach A and only three users exist in the same cluster classified by the human cluster, then the precision is 0.3 , which means only $30 \%$ of the output is correct.

Precision may not be enough for evaluating the performance of an approach, however, because precision only takes the output of an approach into consideration. For example, when all users in a proposed cluster classified by approach A occur in the cluster classified by the human cluster, then the precision is 1 . However, what if we know that there are only three users in the cluster created by approach A but there are twenty users in the cluster classified by the human rater? Approach A no longer appears to be a highly performing technique since it missed 17 of the users as classified by the human rater.

In the abovementioned case, the recall measure provides a means of assessing any misses. The modified definition of the recall measure for this research is

recall $=\frac{\mid \text { manual_cluster_users }|\cap| \text { approach_cluster_users } \mid}{\mid \text { manual_cluster_users } \mid}$.

Take the abovementioned case into consideration, only this time we will use the recall measure to evaluate the performance of approach A. Since there are twenty users in the cluster classified by the human rater and all three users in the proposed cluster classified by approach A are correct, the recall is only 0.15 according to Eq.(2). This is significantly less than the perfect score of 1 for precision.

Once again, the recall measure is not perfect and works independently. For example, if the output of approach A has fifty users in a cluster and the cluster classified by the human rater only has twenty users, then if all of the latter users are in the former group the recall is 1. Clearly, approach $\mathrm{A}$ is not a good approach since 30 users in the cluster should not be so grouped according to the human rater.

It is easy to use precision and recall, but we should not consider only one at a time; that is why we need the Fmeasure [10][17]. The F-measure is a weighted combination of precision and recall and has a range of 0 to 1 .

$F_{\beta}=\left(1+\beta^{2}\right) \times \frac{\text { precision } \times \text { recall }}{\beta^{2} \times \text { precision }+ \text { recall }}$

The F-measure has been used to evaluate the performance of clustering methods [13][21][23]. If a research favours more precision then it sets beta value close to 0.5 (i.e., $F_{0.5}$ ), on the other hand, it sets beta value close to 2 (i.e., $F_{2}$ ) In this research, we also use it to evaluate the performance of the proposed chromosomebased approaches. Table II lists the precision, recall, and Fmeasure values for each approach. 
TABLE II. AVERAGE ${ }^{*}$ PRECISION, RECALL AND F-MEASURE FOR EACH CLUSTERING APPROACH

\begin{tabular}{|r|c|c|c|}
\hline & Standard & Quantitative & $\begin{array}{c}\text { Hierarchical } \\
\text { Clustering }\end{array}$ \\
\hline Precision & $\underline{\mathbf{0 . 7 1 4 6}}$ & 0.7027 & 0.6843 \\
\hline Recall & $\underline{\mathbf{0 . 7 0 6 1}}$ & 0.6747 & 0.6668 \\
\hline F $_{\mathbf{2}}$ & $\underline{\mathbf{0 . 6 9 0 6}}$ & 0.6625 & 0.6513 \\
\hline F $_{\mathbf{0 . 5}}$ & $\underline{\mathbf{0 . 6 9 3 0}}$ & 0.6759 & 0.6590 \\
\hline
\end{tabular}

*. Beside direct use of 2894-bit string chromosomes, we also shorten the chromosome length by considering sentence-based and paragraph-based annotation. We consider a sentence is annotated if the amount of highlighted words in the sentence excesses a certain threshold (percentage), e.g., $10 \%$. We cluster the data set a hundred times with different sentence and paragraph threshold combinations.

The generic hierarchical clustering approach has minimum values of precision and recall compared to the proposed two chromosome-based approaches, but it still has more than $50 \%$ accuracy. It is not good to considering only precision or recall measure for deciding which approach is better as mentioned earlier, so the F-measure values are taken into consideration for the evaluation. According to Table II, the standard approach is the best approach and has maximum values in both $F_{2}$ and $F_{0.5}$ measure, which means that no matter if we favour more precision $\left(\mathrm{F}_{0.5}\right)$ or recall $\left(\mathrm{F}_{2}\right)$ the standard approach is the best one among all approaches.

\section{CONCLUSIONS}

From the results of this research, we can find that the standard approach in student clustering is the best approach, although it produces huge difference values in comparing two chromosomes. Even when we take both speed and performance into consideration, we may still use the standard approach to cluster students according to their annotations, because the standard approach only takes $2.52 \mathrm{~ms}$ more than the quantitative approach and results is slightly superior performance.

With the student clustering results, we can further develop a quick-response recommender system based on the proposed methodology to automatically provide a student the shared thoughts, bookmarks, hot discussions, internal/external references for further readings, and important notes made by other students in the past who belong to the same cluster. Such system can be implemented as a plug-in of learning management systems, e.g., Moodle, and working with annotation plug-in such as Marginalia annotation tool for Moodle. Moreover, such system can work with e-reader applications which allow users making annotations, e.g., Kindle, Apple iBooks app, and Kobo, to provide learners valuable service for selflearning and doing reading activities.

\section{ACKNOWLEDGMENT}

The authors wish to thank the support of Athabasca University, the Mission Critical Research funding, NSERC, iCORE, Xerox and the research related gift funding provided to the Learning Communities Project by Mr. Allan Markin.

\section{REFERENCES}

[1] A. Adler, A. Gujar, B. L. Harrison, K. O'Hara, and A. Sellen, "A diary study of work-related reading: design implications for digital reading devices," Proceedings of the SIGCHI conference on Human factors in computing systems, Los Angeles, California, United States, April 18-23, 1998, pp. 241-248.

[2] E. Ball, H. Franks, J. Jenkins, M. McGrath, and J. Leigh, "Annotation is a valuable tool to enhance learning and assessment in student essays," Nurse Education Today, vol. 29, no. 3, pp. $284-$ $291,2009$.

[3] J. Bien, and R. Tibshirani, "Hierarchical Clustering With Prototypes via Minimax Linkage," Journal of the American Statistical Association, in press. Retrieved on December 30, 2011, from http://www-stat.stanford.edu/ jbien/jasa2011minimax.pdf

[4] C.-K. Chang, G.-D. Chen, and C.-K. Chen, "Using computer-based annotation to promote the summary of reading comprehension," Communication of IICM, vol. 9, no. 1, pp. 41-58, 2006.

[5] P.-Y. Chao, G.-D. Chen, and C.-W. Chang, "Developing a Crossmedia System to Facilitate Question-Driven Digital Annotations on Paper Textbooks," Journal of Educational Technology \& Society, vol. 13 , no. 4, pp. 38-49, 2010.

[6] A. F. Chiarella, "Enabling the collective to assist the individual: A self-organising systems approach to social software and the creation of collaborative text signals," Unpublished PhD Thesis, Montreal, McGill University, 2008.

[7] A. F. Chiarella, and S. P. Lajoie, "A self-organising systems approach to history-enriched digital objects,". In D. Ifenthaler, P. Pirnay-Dummer \& N. M. Seel (Eds.), Computer-Based Diagnostics and Systematic Analysis of Knowledge, pp. 131-158, 2010, Boston, MA: Springer.

[8] X. Cui, J. Gao, and T. E. Potok, "A flocking based algorithm for document clustering analysis," Journal of Systems Architecture, vol. 52, no. 8-9, pp. 505-515, 2006.

[9] C. Ding, and T. Li, "Adaptive dimension reduction using discriminant analysis and K-means clustering," Proceedings of the 24th international conference on Machine learning (ICML 2007), Corvallis, Oregon, United States, June 20-24, 2007. Retrieved on December 30, 2011, from http://www.machinelearning.org/proceedings/icml2007/papers/521 .pdf

[10] G. Hripcsak, and A. S. Rothschild, "Agreement, the F-Measure, and Reliability in Information Retrieval," Journal of the American Medical Informatics Association, vol. 12, no. 3, pp. 296-298, 2005.

[11] W.-Y. Hwang, and G.-L. Hsu, "The effects of pre-reading and sharing mechanism with annotations on learning," The Turkish Online Journal of Educational Technology, vol. 10, no. 2, pp. 234249, 2011.

[12] W.-Y. Hwang, C.-Y. Wang, M. Sharples, "A study of multimedia annotation of Web-based materials," Computers \& Education, vol. 48, no. 4, pp. 680-699, 2007.

[13] A. K. Jain, "Data clustering: 50 years beyond K-means," Pattern Recognition Letters, vol. 31, no. 8, pp. 651-666, 2010.

[14] L. Khan, M. Awad, and B. Thuraisingham, "A new intrusion detection system using support vector machines and hierarchical clustering," The International Journal on Very Large Data Bases, vol. 16, no. 4, pp. 507-521, 2007.

[15] J. Kurhila, M. Miettinen, P. Nokelainen, P. Floréen, and H. Tirri, "Peer-to-Peer Learning with Open-Ended Writable Web," Proceedings of the 8th Annual Conference on Innovation and Technology in Computer Science Education (ITiCSE 2003), Thessaloniki, Greece, June 30 - July 02, 2003, pp. 173-177.

[16] M.-J. Li, M.-K. Ng, Y.-M. Cheung, and L.-Z. Huang, "Agglomerative Fuzzy K-Means Clustering Algorithm with Selection of Number of Clusters," IEEE Transactions on Knowledge and Data Engineering, vol. 20, no. 11, pp. 1519-1534, 2008.

[17] D. D. Lewis, and W. A. Gale, "A sequential algorithm for training text classifiers," Proceedings of the 17th annual international ACM SIGIR conference on Research and development in information retrieval (SIGIR 1994), Dublin, Ireland, July 03-06, 1994, pp.3-12. 
[18] C. C. Marshall, "Annotation: from paper books to the digital library," Proceedings of the second ACM international conference on Digital Libraries, 1997, pp. 131-140.

[19] U. Neisser, "Rising scores on intelligence tests," American Scientist, vol. 85, no. 5, pp. 440-447, 1997. Retrieved on Decembe 30, 2011, from http://www.americanscientist.org/issues/pub/risingscores-on-intelligence-tests/1

[20] K. O'Hara, and A. Sellen, "A Comparison of Reading Paper and On-Line Documents," Proceedings of the SIGCHI conference on Human factors in computing systems, Atlanta, Georgia, United States, March 22-27, 1997, pp. 335-342

[21] M. Rasmussen, and G. Karypis, "gcluto: An interactive clustering, visualization, and analysis system," Technical Report CSE/UMN
TR 04-021, Department of Computer Science and Engineering, University of Minnesota, 2004.

[22] P.-L. P. Rau, S.-H. Chen, and Y.-T. Chin, "Developing web annotation tools for learners and instructors," Interacting with Computers, vol. 16, no. 2, pp. 163-181, 2004.

[23] M. Steinbach, G. Karypis, V. Kumar, "A Comparison of Document Clustering Techniques," Proceedings of KDD-2000 Workshop on Text Mining, Boston, MA, USA, August 20, 2000.

[24] R. Xu, and D. Wunsch, II, "Survey of clustering algorithms", IEEE Transactions on Neural Networks, vol. 16, no. 3, pp. 645-678, 2005

[25] S.-W. Yeh, and J.-J. Lo, "Using online annotations to support error correction and corrective feedback," Computer \& Education, vol 52, no. 4, pp. 882-892, 2009. 